\title{
CONTROL OF LARGE FLEXIBLE SPACE STRUCTURES
}

\author{
Semi-Annual Status Report \\ for the period \\ November 16, 1985 to May 15, 1986
}

NASA Research Grant No. NAG1-126

Principal Investigator: Prof. W.E. Vander Velde Department of Aeronautics and Astronautics

Massachusetts Institute of Technology. Cambridge, MA 02139

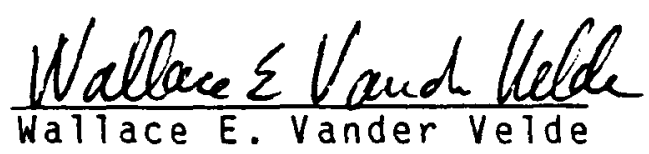

$$
\text { JuTy } 23,1986
$$

(NASA-CR-177063) CCNTBOL OE IARGE FLEXIBLE SEACE SIRUCTURES Semiannual status Report, 16 Nov. 1985 - 15 May 1986 (Massachusetts Inst. of Tech.) $11 \mathrm{p}$ 


\section{CONTROL OF LARGE FLEXIBLE SPACE STRUCTURES}

\section{Introduction}

This is the report of the status of work under NASA Research Grant No. NAGI-126 for the period November 16, 1985, to May 15, 1986. This grant was initiated a number of years ago with the charter to concentrate on the problem of component unreliability in control systems for large flexible space structures. That focus was maintained until this reporting period when the charter was broadened to include other aspects of controlling flexible spacecraft as well. Reflecting the broader scope of the program, a new research task was undertaken to study the design and analysis of control systems for space structures with joints having nonlinear properties.

The immediate motivation for the work on control systems for joint-dominated structures is the COFS program where the requirement to deploy and retract the test structure rules out the use of positive-locking joints. The baseline joint design for this structure displays distinctly nonlinear behavior, and this poses a serious challenge to all experimental work with that structure - including system identification, control system design, and failure detection. It is hoped that the background we develop in addressing the nonlinear joint problem can be put to use in the COFS program along with the extensive background we have in the FDI area.

\section{Research Progress}

During this reporting period we had activity on four research tasks: robust design of generalized parity relations, design of failure sensitive observers using the geometric system theory of Wonham, computational techniques for evaluation of the performance of control systems with fault tolerance and redundancy management features, and the design and evaluation of control systems for structures having nonlinear joints. A brief report on each of these research areas follows.

Robust design of generalized parity relations - Our work on this subject was largely completed during the prior reporting period, but the thesis of Jean Dutilloy which summarizes this work was published during the past six months. Early in this research program we had decided to concentrate attention on two methods of residual generation for failure monitoring - the failure detection filter and generalized parity relations. These methods were selected because they appear to be the only methods whtch have been suggested which have the desirable properties of being applicable to both sensors and actuators and of not requiring hypotheses about the mode of component 
failures. Our objective was to study both methods carefully, especially with a view to making them as useful as possible in the presence of system dynamics modeling errors. Only when both approaches are utilized to their best advantage would it be fair to compare them, both in simulation and experimentally, and draw conclusions about their relative utility.

A previously-submitted report by Alejandro San Martin summarizes our work on robust design of failure detection filters as Dutilloy's report does for generalized parity relations. He tested two approaches to estimation of the coefficients of the parity relations based on data recorded from the actual structure in flight. One approach implemented the kalman filter to estimate the coefficients. This met with some success in improving the performance of the residual generator when the dynamics of the structure were somewhat different from the model available before flight, but the procedure is very demanding of computational resources. An alternative procedure which is less intensive computationally was also investigated. This is essentially a gradient search for the set of coefficients which minimize the mean squared residuals in the absence of failure - with just one gradient step taken with each new sample of data. As should be expected, this method did not work as well as the kalman filter although it did reduce the residuals due to missmodeled dynamics.

The most promising approaches appear to be methods of constructing the parity relations directly from input-output data collected in flight. These approaches are actually independent of any a priori model of the spacecraft dynamics, so they avoid altogether the question of how well predicted the dynamic properties of the structure will be in advance of flight. The best formulation of this problem is to compute the parity relation coefficients which maximize the ratio of RMS failure signature to RMS noise in the absence of failures. Once a suitably long sample of data is collected, the solution depends on the eigenvector decomposition of a high dimension matrix. This is a substantial computational requirement, but there is no real-time constraint on the procedure. Parity relations constructed in this way could accommodate any magnitude of errors in the parameters of the dynamic model of the system. However, since the length of the parity relation is fixed at a chosen value, it is sensitive to spillover from higher order modes which cannot be represented in a parity relation of the chosen length. And, of course, the method depends on linearity of the system dynamics.

We are now at a point where we could usefully compare the performance of failure detection filters and generalized parity relations in an experimental setting - perhaps on the grillage apparatus at Langley Research Center. 
Failure sensitive observers via geometric system theory This Tine of research has been pursued by Mohammad Massoumnia who has been a Doctoral candidate in the Department of Aeronautics and Astronautics. He completed his thes is during this reporting period and the report of his work has been submitted formally to NASA. Several papers have been prepared by Mohammad, in some cases with coauthors, and have been submitted to journals.

This is an outstanding piece of research. The geometric theory was found to be a very natural and convenient approach to the characterization of observers which restrict the signatures of component failures to separate subspaces so the faulty component can be isolated without consideration of mode of failure. Some solutions for such observers were found for cases where the failure detection filter as defined by Beard has no solution. It was found that observers of reduced order can often be used as opposed to the full order structure of the failure detection filter. With state augmentation, it was found that the signatures of sensor failures can be restricted to a single line in the output space whereas the failure detection filter can only restrict such signatures to a plane. The theory also leads quite naturally to design methodologies for which stable, modern numerical algorithms exist.

When the FDI problem is formulated as a general observer problem, with the inputs being the measurements and the actuator commands and the outputs being the residuals which are to be monitored for failure signatures, and set in discrete time, the geometric theory leads to solutions which allow freedom to place the observer poles arbitrarily. If the poles are all placed at the origin, one gets a finite impulse response filter which is a generalized parity relation. Alternatively, one can insist upon a model-based observer structure, in which case a discrete time failure detection filter results. So these two seemingly different approaches to FDI residual generation are seen to be special cases of more general failure sensitive observers. It is true, however, that a discrete time filter cannot hold failure signatures strictly in separate subspaces if the failure mode is not constant between samples - which is almost always the case. The error is small according to how fast the data are sampled relative to the frequency content of the sensor outputs, the actuator commands, and the actual output of the failed component.

As this work appears in the professional journals, it will constitute a very important addition to the literature on failure detection in linear systems. 
System performance evaluation - Previous status reports have introduced the concept of evaluating the system performance by calculating the system unreliability and by finding the probability mass function (pmf) of the value of a system performance measure. The performance is dependent on the time-varying status of the fault-tolerant system. The system status changes as failures and fault detection decisions occur. These status changes are modelled by a finite state Markov process in order to execute the desired calculations. It is assumed that the performance measure for the system depends upon the trajectory of the system status process, thus it is a random variable that obeys some pmf, and the objective of our research is to find this pmf.

Previous status reports have also discussed the primary difficulty with the conceptual method outined above. In practice, the number of possible trajectories for the system status is unmanageably large. This provided the motivation for the "v-transform" method of calculating the performance pmf introduced in [1]. In the status report for November, 1985, the "z-transform" method was described as part of an alternative approach to the one presented in [1]. The primary advantage of the $z$-transform method resided in the fact that, in order to find the moments of the system performance pmf, the single shtep $v$-transform matrix $M(v)$ did not have to be raised to the $k$ th
power, as it did in [1]. Recall that each element of $M(v)$ is a power, as it did in [1]. Recall that each element of M(v) mission and is therefore an integer that could be very large. The z-transform method yielded good results. However, its application assumes that the matrix $M_{0}$ (the zeroth order term of $M(v))$ is diagonalizable with distinct eigenvalues. Moreover, some accuracy is lost during the calculations because of the eigenstructure decomposition that is performed as part of the method.

The "s-transform" has been developed as a new method for performance pmf evaluation that eliminates the drawbacks but retains the advantages of the "z-transform" approach.

Furthermore, this new alternative requires approximately the same amount of CPU time and memory as the former one.

The "s-transform" is defined as:

$$
m_{i j}(s)=p_{i j} \exp \left(J_{i} s\right)
$$

where $p_{i j}$ is the single step probability of transition from state $j j_{0}$ state $i$, and $J j$ is the performance value associated with occupying state $i$. Then, if the $m_{j}$ are defined to be the elements of the single step s-transformmatrix $M(s)$, the corresponding element of $M^{R}(s)$ takes the form:

$$
m_{i j}(k)=\sum_{\ell} \bar{p}_{i j}(\ell) \exp \left(s j^{\prime}(\ell)\right)
$$


where the l-summation is over all the distinct trajectories going from state $j$ to state $i$ in $k$ time steps, $p^{\prime} j(l)$ is the probability of the $\ell^{t h}$ trajectpry, and $J^{\prime}(l)$ is the performance value accumulated along the $e^{t h}$ trajectory. By using a truncated series expansion of the exponential, each $m_{i j}(s)$ can be expressed as:

$$
m_{i j}=p_{i j}+p_{i j} J_{i} s+\ldots+p_{i j} J_{i}^{n} s^{n} / n !
$$

where $n$ is the $h$ ighest order of the moments of the performance pmf to be matched. Therefore, $M(s)$ can be written as:

$$
M(s)=M_{0}+M_{1} s+\ldots+M_{n} s^{n} / n !
$$

If the initial state probability vector $\pi(0)$ is known, then it is possible to evaluate the vector (dependent on the terminal state) of $s$-transforms for a $k$-step mission $\pi(k, s)$ as:

$$
\pi(k, s)=M^{k}(s) \pi(0)
$$

which can be written as:

$$
\pi(k, s)=N_{0}+N_{1} s+\ldots+N_{n} s^{n} / n !
$$

where the $N_{j}$ are the vectors of $j^{\text {th }}$ order moments of the performancejvalue given the terminal state. Using this method, any number of moments of the performance value may be calculated for each terminal state without committing any of the numerical error due to the decomposition routines that were used by the "z-transform" method.

With knowledge of these moments, the performance pmf can be approximated by a distribution of the form:

$$
P(x)=\exp \left(A_{0}+A_{1} x+\ldots+A_{n} x^{n}\right)
$$

where the $A$ are to be determined such that the first $n+1$ moments of the performance value that were calculated above are matched. The status report of November, 1985, discussed this approximation and some of the results that had been obtained up to that time. Since then, the Newton-Raphson routine that is used to find the $A$ has been considerably modified. Almost any performance pmf may now be approximated by the exponential form above with an exponent polynomial of degree 7 or 9 and the Newton-Raphson coefficient calculation routine will converge.

This method gives very good approximations to the performance pmf's that have been examined so far. Consider, for instance, the 7-state example developed in [1]. Figures 1 and 2 present the performance pmf for this 7-state model for a mission duration of 750 time steps. Figure 1 was obtained with the 
method developed in [1] while Figure 2 was generated using the new approach. Figure 4 shows the pmf for a mission duration of only 150 time steps calculated by the new approach. By comparing it to the corresponding result obtained in [1] (shown in Figure 3), we notice that details like the spike at the origin and the plateau are reproduced because of the decomposition of the performance pmf according to the terminal state that the new method employs. It is important to note that the new method was executed in FORTRAN on an IBM personal Computer AT while the results in [1] were generated by a LISP machine attached to a very large memory network. This makes the close agreement between the results quite remarkable.

Despite the apparent close agreement of the results using the new method and the results of [1], it is still true that the number of coefficients $A_{i}$ that are included markedly influences the shape of the tail of the distribution. This is evident in Figures 5 and 6 , which represent the approximation with 7 and 9 coefficients, respectively, to the pmf of the 7-state model for a mission duration of 750 time steps. This is the same case that is depicted in Figures 1 and 2 except that the horizontal scale has been reduced so that the tail of the pmf becomes visible. Note from Figure 1 that the method of [1] produces only a truncated version of the performance pmf (despite its heavy use of computational resources) while the new method produces an approximation to the pmf that is valid over any desired range of performance values.

Present1y, we are working on a practical example of performance evaluation for a realistic fault-tolerant system. In the absence of an architecture for the redundancy management of a LSS, we have begun to examine the performance, measured by the terminal orbit insertion accuracy, of the IUS navigation system for a typical flight mission. This will demonstrate the applicability of our techniques to the complex models that might be expected for a LSS redundancy management system.

Control of joint-dominated structures - This is a new research task initiated during the present reporting period. The motivation for this work is the prototype design of the cofs experimental structures. The MAST is to be deployed from the cargo bay of the space shuttle, and retracted again for use on future missions. Due to its erectable design, it contains many joints - and because it must be retracted for reuse, the joints cannot have positive locking properties when erected. Partly for this reason, the baseline joint design displays a distinctly nonlinear character under test. Probably due to a small amount of play between the pins and the holes, the joint has a strongly hardening spring character with hysteresis. Almost all of the work on control of large space structures, including identification and FoI as well as control, has been done in the context of linear models of the structurai dynamics. It is a 
considerable step to carry these technologies into the domain of nonlinear plant dynamics.

We are looking first at the design and evaluation of control systems for structures with nonlinear joints. It is expected that some of the methodologies developed in the control context will have some application to identification and failure detection as well. It seems embarassing that the control community has so few tools to apply to the design of control systems for nonlinear plants of higher order than two - for which the phase plane methodology is very useful. Some of the more elegant mathematical techniques, such as functional series expansion and catastrophe theory, are not applicable to nonlinearities with multivalued characteristics - such as the hysteretic characteristic. A survey of the literature on nonlinear system theory turned up no methodologies that seem applicable to high order systems with multivalued nonlinearities other than describing function theory - which in principle can be used to test for limit cycles, determine response to sinusoidal inputs, and evaluate response to random inputs. The applicability of describing function theory to large space structures must be qualified by the fact that this approach generally works well only when the plant has a low pass character. This is because, for sinusoidal analysis, the higher harmonics are assumed attenuated by the plant, and for random response analysis, the inputs to the nonlinearities are assumed gaussian - and this tends to be a good approximation with low pass plants. The resonant character of structures in space may present a problem in this respect.

Our plan is to use sinusoidal-input describing functions to test a proposed control system design for limit cycles. It is certainly to be desired that the system not limit cycle. If that is shown to be the case, then random-input describing functions are to be used to evaluate the performance of the control system in regulating against random disturbances. This second step presents a challenge in that random-input describing functions as usually formulated do not apply to multivalued nonlinearities. However, there is an alternate formulation which utilizes the expansion of gaussian processes into a series of sinusoids and allows use of the sinusoidal-input describing function to characterize the nonlinearities. This approach is applicable to multivalued nonlinearities.

Our initial effort, after an extensive study of alternative approaches, has been directed toward the search for 1 imit cycles in systems with low-ordered resonant plants. Joint characteristics similar to those recorded in tests of the baseline joint are modeled. In a multi-nonlinearity setting, this requires a search for the frequency of the 1 imit cycle and the amplitudes of all the variables at the inputs to the nonlinearities such that the conditions for a sustained 
oscillation are satisfied. Our experience so far is that the search is not well behaved. Depending on the choice of starting point, the search can converge to the solution or may drift off to very large values of the solution variables. We are presentiy surveying the nature of the function whose zero value indicates a limit cycle to see if we can predict starting points which will give convergence. Eventually, even is this succeeds, we will have to compare the computational burden of searching for limit cyles using describing function modeling versus testing for limit cyles by simulating the system response from a number of initial states.

Terminal equipment for communication with LaRC - During this reporting period the project acquired some terminal equipment for the purpose of remote control of experiments at LaRC. The equipment includes an IBM PC/XT with $640 \mathrm{~kb}$ of memory, a monochrome monitor, and a graphic monitor adaptor. We also have a modem capable of operation up to 2400 baud and a software package - Cross Talk - which implements terminal emulation and file communication. It is our intent to use this capability to test FDI algorithms on the grillage apparatus starting in the fall, and later to use the same approach with the COFS equipment - especially the MINIMAST. It is appreciated that remote operation of experiments at LaRC requires the time and attention of personnel at LaRC as well, and this will have to be negotiated.

\section{Personnel}

The Principal Investigator for this research program is Professor Wallace E. Vander Velde of the MIT Department of Aeronautics and Astronautics. He devoted $20 \%$ of $h$ is time to this program and supervised the work of the following students:

Jean Dutilloy - He is a Masters degree candidate who finished his work near the beginning of this reporting period. He worked on the robust design of generalized parity relations. His thes is has been submitted

formally as a report to NASA. He had a fellowship and derived only computational support from the grant.

Mohammad Massoumnia - He is a doctoral candidate who finished his work during this reporting period. He was a Research Assistant supported by the grant. He worked on the application of geometric system theory to the design and interpretation of failure-sensitive observers. His thes is was submitted formally as a report to NASA and several papers have been submitted to professional journals for publication. 
Mathieu Mercadal - He is a masters candidate who joined this program as a Research Assistant last fall. He initiated the research task on control of joint-dominated structures. He is a very bright student who has passed the Qualifying Exam for the doctoral program - recording the highest score in the Department on that examination.

Another faculty member associated with this program is Professor Bruce $K$. Walker, also of the MIT Department of Aeronautics and Astronautics. He devoted about 10 percent of his time to this effort and supervised the work of the following student:

Jean-0livier Missana - He is a graduate student Research Assistant supported by the program. He is a candidate for the Masters degree and has been working on the system performance evaluation task.

Publications and Presentations

Dutilloy, Jean R.: "Generalized Parity Relations for Large Space Structures with Uncertain Parameters", SSL Report No. 2-86, January 1986. This report was submitted formally to NASA.

Massoumnia, Mohammad A.: "A Geometric Approach to Failure Detection and Identification in Linear Systems", SSL Report No. 5-86, February 1986. This report was submitted formally to NASA.

\section{Reference}

[1] Gerber, D.K., Performance evaluation of fault-tolerant systems using transient Markor models, S.M. Thesis, Dept. of Aeronautics and Astronautics, M.I.T., Cambridge, MA, April 1985 . 


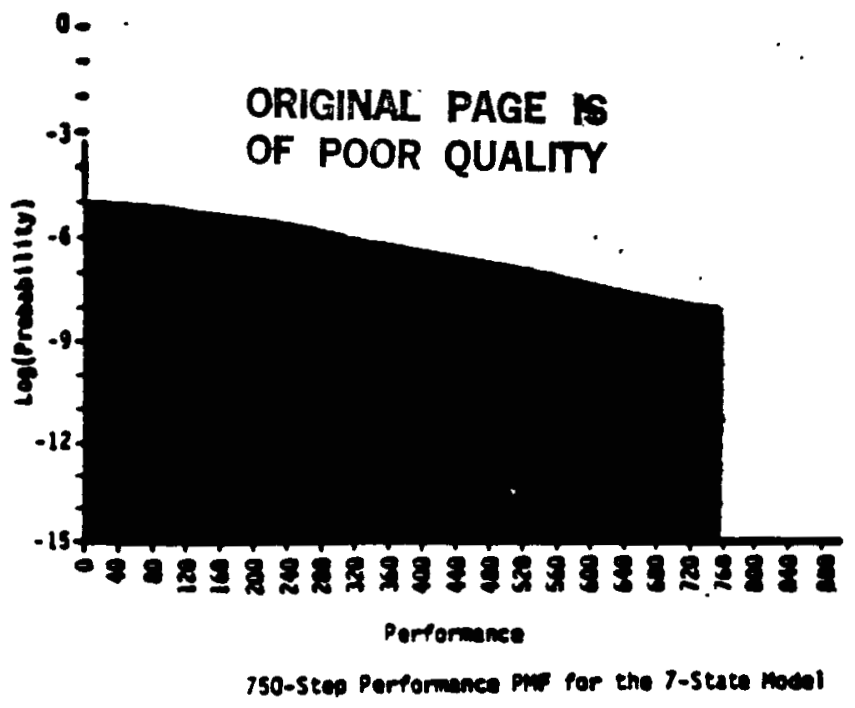

Figure 1

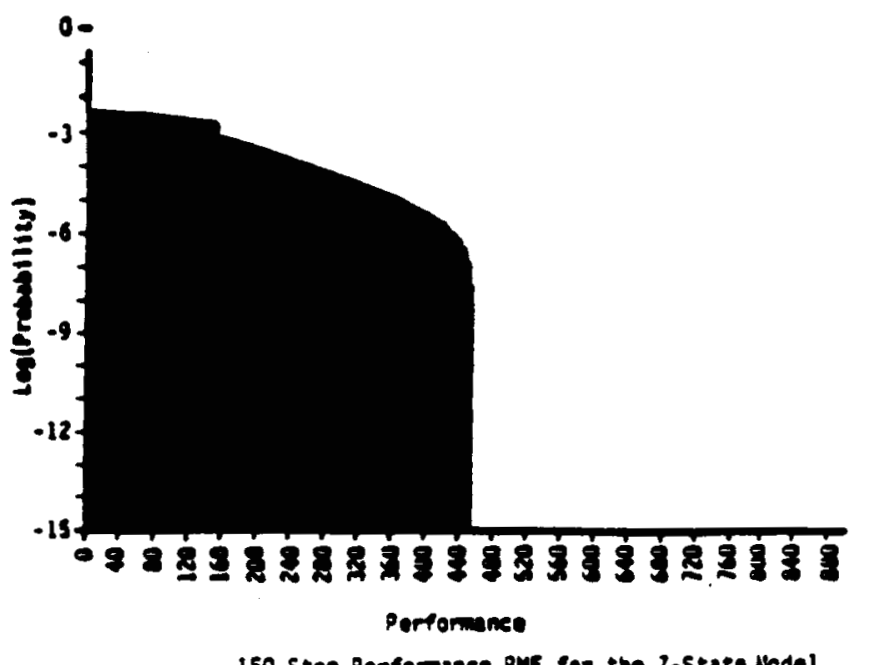

Figure 3

7 STATE MOOEL

750 TIME SIEPS, 7 COEFFICIENTS, ACCURACY : I.E-8

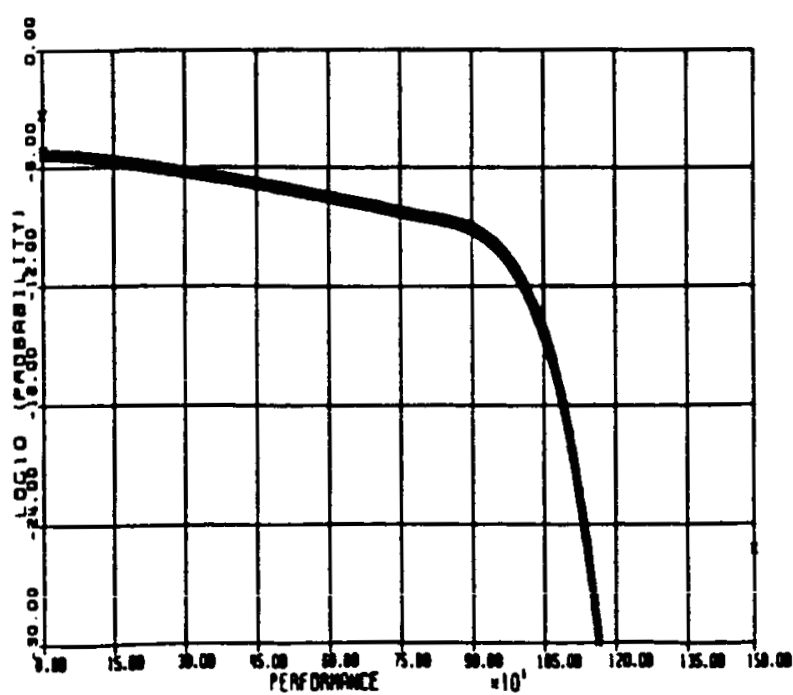

Figure 5
7 STATE MODEL

750 TIME STEPS. 9 COEFFICIENTS. RCCURACY : I.E-8

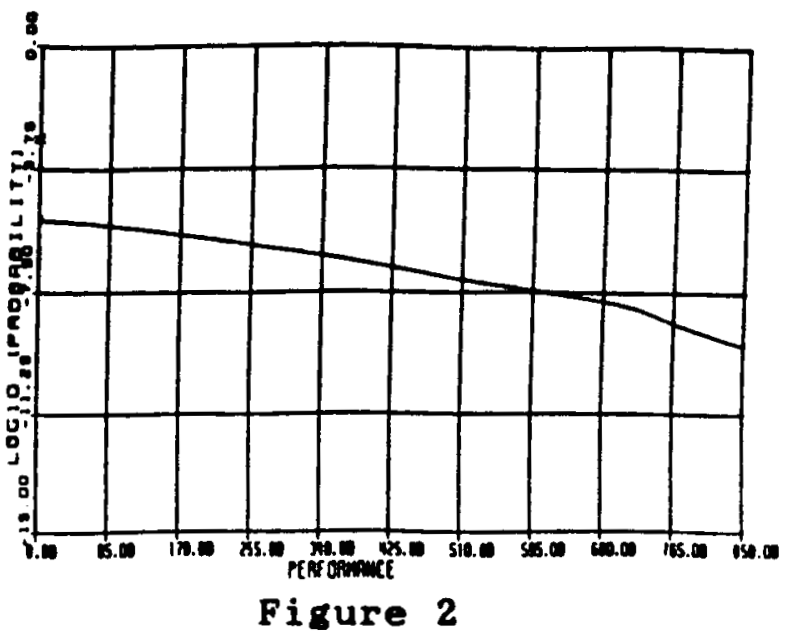

7 STATE MOOEL

150 IIME SIEPS. 7 COEFFICIENTS. ACCURACY : I.E-B

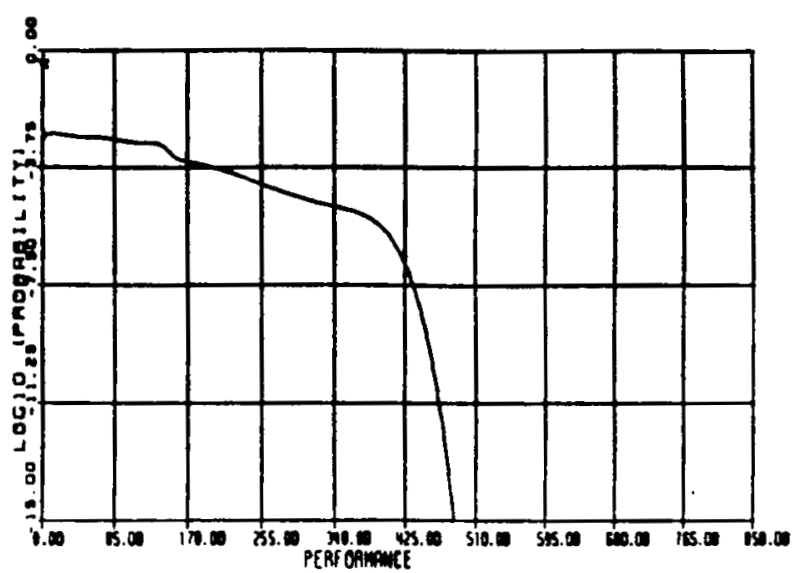

Figure 4

7 STATE MOOEL

750 TIHE SIEPS, 9 COEFFICIENIS, ACCURACY : I.E-8

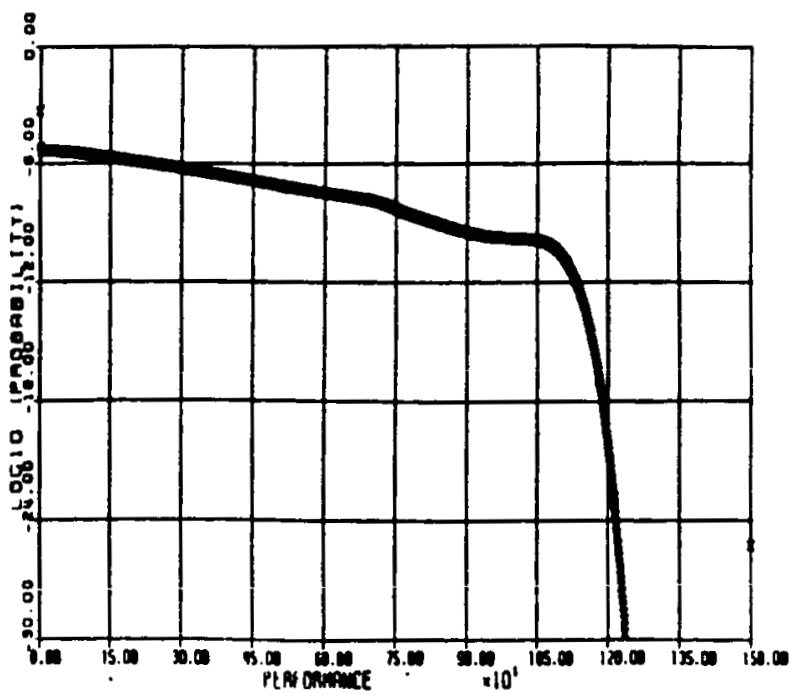

Figure 6 